\title{
Editorial
}

\section{Computational Intelligence in Image Processing}

\author{
Erik Cuevas, ${ }^{1}$ Daniel Zaldívar, ${ }^{1}$ Gonzalo Pajares, ${ }^{2}$ Marco Perez-Cisneros, ${ }^{1}$ and Raul Rojas ${ }^{3}$ \\ ${ }^{1}$ Departamento de Ciencias Computacionales, Universidad de Guadalajara, CUCEI, Avenida Revolución 1500, \\ Guadalajara, Jal, Mexico \\ ${ }^{2}$ Departamento de Ingeniería de Software e Inteligencia Artificial, Facultad Informática, Universidad Complutense, \\ 28040 Madrid, Spain \\ ${ }^{3}$ Institut für Informatik, Freie Univerisität Berlin, Arnimallee 7, 14195 Berlin, Germany \\ Correspondence should be addressed to Erik Cuevas; erik.cuevas@cucei.udg.mx
}

Received 20 November 2013; Accepted 20 November 2013

Copyright (C) 2013 Erik Cuevas et al. This is an open access article distributed under the Creative Commons Attribution License, which permits unrestricted use, distribution, and reproduction in any medium, provided the original work is properly cited.

Vision in general and images in particular have always played an essential role in human life. In the past they were, today they are, and in the future they will continue to be one of our most important information carriers. Recent advances in digital imaging and computer hardware technology have led to an explosion in the use of digital images in a variety of scientific and engineering applications. These applications result from the interaction between fundamental scientific research on the one hand and the development of new and high-standard technology on the other.

Computational intelligence has emerged as a powerful tool for information processing, decision making, and knowledge management. The techniques of computational intelligence have been successfully developed in areas such as neural networks, fuzzy systems, and evolutionary algorithms. It is predictable that in the near future computational intelligence will play a more important role in tackling several engineering problems.

Image processing is a very important research area. Classical image processing methods often face great difficulties while dealing with images containing noise and distortions. Under such conditions, the use of computational intelligence approaches has been recently extended to address challenging real-world image processing problems. The interest on the subject among researchers and developers is increasing day by day as it is branded by huge volumes of research works that get published in leading international journals and international conference proceedings.

When the idea of this special issue was first conceived, the goal was to mainly expose the readers to the cutting-edge research and applications that are going on across the domain of image processing where contemporary computational intelligence techniques can be or have been successfully employed.

The special issue received 49 high-quality submissions from different countries all over the world. All submitted papers followed the same standard of peer-review by at least three independent reviewers, as it is applied to regular submissions to Mathematical Problems in Engineering. Due to the limited space, 16 papers were finally included. The primary guideline was to demonstrate the wide scope of computational intelligence algorithms and their applications to image processing problems.

The paper authored by K. haransiriphaisan et al. presents a study of the performance of a family of artificial bee colony algorithms, namely, the standard $\mathrm{ABC}, \mathrm{ABC} /$ best/1, $\mathrm{ABC} /$ best $/ 2, \mathrm{IABC} /$ best $/ 1, \mathrm{IABC} / \mathrm{rand} / 1$, and $\mathrm{CABC}$ and some particle swarm optimization-based algorithms for searching multilevel thresholding in image segmentation. The strategy for an onlooker bee to select an employee bee was modified for a new alternative mechanism. The experimental results showed that IABC/best/ 1 outperformed the other techniques, when all of those algorithms were applied for multilevel image segmentation.

The paper by B. Ojeda-Magaña et al. proposed the concept of typicality from the field of cognitive psychology to categorize data into concepts. The idea is to apply such concepts into the interpretation of numerical data in color images for segmentation purposes.

S. E. Gonzalez-Reyna et al. propose a road sign detection method based on oriented gradient maps and the KarhunenLoeve transform. The proposed algorithm is able to reach 
good classification accuracy by using a reduced amount of attributes compared to other similar state-of-the-artmethods.

I. Cruz-Aceves et al. present an automatic image segmentation method based on the theory of active contour models and estimation of distribution algorithms. The proposed method uses the univariate marginal distribution model to infer statistical dependencies between the control points on different active contours. These contours have been generated through an alignment process of reference shape priors aiming to increase the exploration and exploitation capabilities regarding different interactive segmentation techniques. The algorithm is applied in the segmentation of images with different complexity levels.

The paper by S.-F. Tu and C.-S. Hsu proposed the use of a modified version of the popular differential evolution (DE) to construct an optimal least Significant Bit (LSB) matrix for information hiding in images.

The paper authored by L. Shen et al. presents a robust Bayesian approach for multisensor image matching. The method is implemented by using the gradient prior information which is estimate by the kernel density estimated (KDE) method, whereas the likelihood is modeled according to the distance of orientations. Experiments on several groups of multisensor images show that the proposed method outperforms the standard methods in terms of robustness and accuracy.

V. Magudeeswaran and C. G. Ravichandran introduce a new method for histogram equalization based on fuzzy logic. The method consists of two stages. First, the fuzzy histogram is computed based on fuzzy set theory to handle the inexactness of gray level values. In the second stage, the fuzzy histogram is divided into two subhistograms based on the median value of the original image and then equalizes them independently to preserve image brightness. The performance of the proposed approach was evaluated considering qualitative and quantitative performance indexes.

$\mathrm{S}$. Chen et al. present a universal noise removal algorithm by combining spatial gradient and a new impulse statistic into the trilateral filter. Simulation results show that the proposed method has a high denoising rate, in particular for salt-andpepper noise. It is also demonstrated that the computational complexity of the proposed method is less than many other mixed noise filters.

The paper by Y. R. Tabar and I. Ulusoy presented a study where several segmentation methods are implemented and applied to segment the facial masseter tissue from magnetic resonance images. In the study, a new approach is also proposed where unlabeled prior information from a set of MR images is used to segment masseter tissue within a probabilistic framework. The proposed method uses only a seed point that indicates the target tissue and performs automatic segmentation for the selected tissue avoiding the use of a labeled training set. The segmentation results of all methods are validated and compared to particularly discuss the influences of labeled or unlabeled prior information and initialization.

I.-D. Lee et al. present a reversible data hiding scheme to embed secret data into a side matched vector quantization (SMVQ)-compressed image. By using this strategy, fewer bits can be utilized to encode SMVQ indexes with very small values. Experimental results show that the performance of the proposed scheme is superior to those from former data hiding schemes for VQ-based, VQ/SMVQ-based, and search order coding (SOC)-based compressed images.

P. Han et al. present a computational intelligence method by using wavelet optical flow and hybrid linear-nonlinear classifier for object detection. The algorithm can accurately detect moving objects with variable speeds in a scene. Experimental results confirm that the proposed object detection method possesses an improved accuracy and computation efficiency over other state-of-the-art methods.

The paper by Z. Fan et al. proposes a filter algorithm that combines multi-objective genetic algorithm (MOGA) and shearlet transformation to remove noise in images. The approach considers five stages. First, the multiscale wavelet decomposition is applied to the target image. Second, the MOGA target function is constructed by evaluation methods, such as signal to noise ratio (SNR) and mean square errors (MSE). Then, MOGA is used to calculate optimal coefficients of shearlet wavelet threshold value in different scales and different orientations. Finally, the noise-free image is obtained through inverse wavelet transform. Experimental simulations show that the proposed algorithm eliminates noise more effectively and yields better peak signal noise ratio (PSNR) gains compared to other traditional filters.

C. A. Atoche et al. present a parallel tool for largescale image enhancement/reconstruction and postprocessing for Radar/SAR sensor systems. The proposed parallel tool performs the following intelligent processing steps: image formation for the application of different system-level effects of image degradation with a particular remote sensing (RS) system and simulation of random noising effects, enhancement/reconstruction by employing nonparametric robust high-resolution techniques, and image post processing using the fuzzy anisotropic diffusion technique which incorporates a better edge-preserving noise removal effect and faster diffusion process. To verify the performance implementation of the proposed parallel framework, the processing steps were developed and specifically tested on graphic processor units (GPUs).

The paper authored by E. Cuevas et al. presents an algorithm for the automatic detection of circular shapes from complicated and noisy images with no consideration of the conventional Hough transform principles. The proposed algorithm is based on a newly developed evolutionary algorithm called the adaptive population with reduced evaluations (APRE). The approach reduces the number of function evaluations through the use of two mechanisms: (1) adapting dynamically the size of the population and (2) incorporating a fitness calculation strategy which decides whether the calculation or estimation of the new generated individuals is feasible. As a result, the method can substantially reduce the number of function evaluations, yet preserving the good search capabilities of an evolutionary approach. Experimental results over several synthetic and natural images, with a varying range of complexity, validate the efficiency of the proposed technique with regard to accuracy, speed, and robustness. 
B.-Y Park et al. propose a multilabel segmentation that aims to divide a texture image into multiple regions based on a homogeneity condition using local entropy measured at varying scales. For multi-label segmentation, a bipartitioning segmentation scheme is recursively applied to confined regions obtained by previous segmentation steps. On the other hand, the entropy is employed to determine the spatial regularity of elementary texture structures. Experimental results on a variety of texture images demonstrate the efficiency and robustness of the proposed algorithm.

The paper by Haisheng Song et al. presented a neural network (NN) system to classify remote sensing images. The approach uses genetic algorithm to generate the initial structure of NN. Then, weights adjustments are provided by the traditional backpropagation algorithm. Finally, a hybrid algorithm is used to execute classification on remote sensing images. Results show that the hybrid algorithm outperforms other similar approaches.

\section{Acknowledgments}

Finally, we would like to express our gratitude to all of the authors for their contributions and the reviewers for their effort providing valuable comments and feedback. We hope this special issue offers a comprehensive and timely view of the area of applications of computational intelligence in image processing and that it will grant stimulation for further research.

Erik Cuevas

Daniel Zaldivar

Gonzalo Pajares

Marco Perez-Cisneros

Raul Rojas 


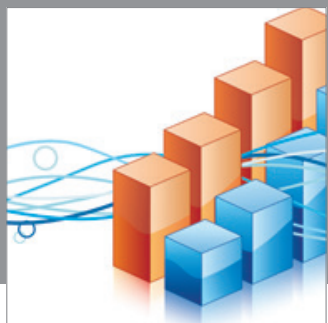

Advances in

Operations Research

mansans

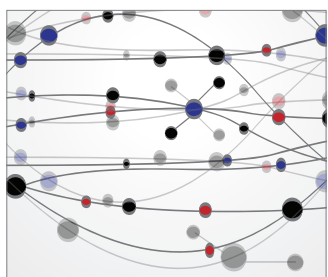

The Scientific World Journal
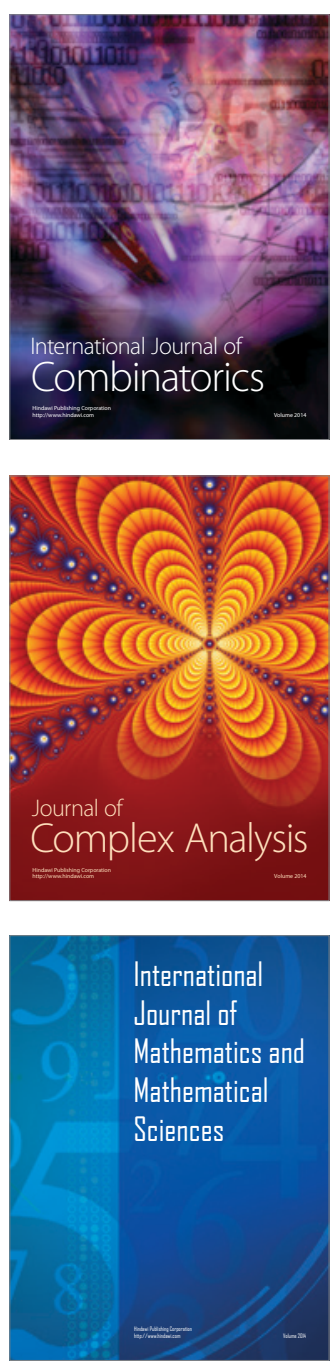
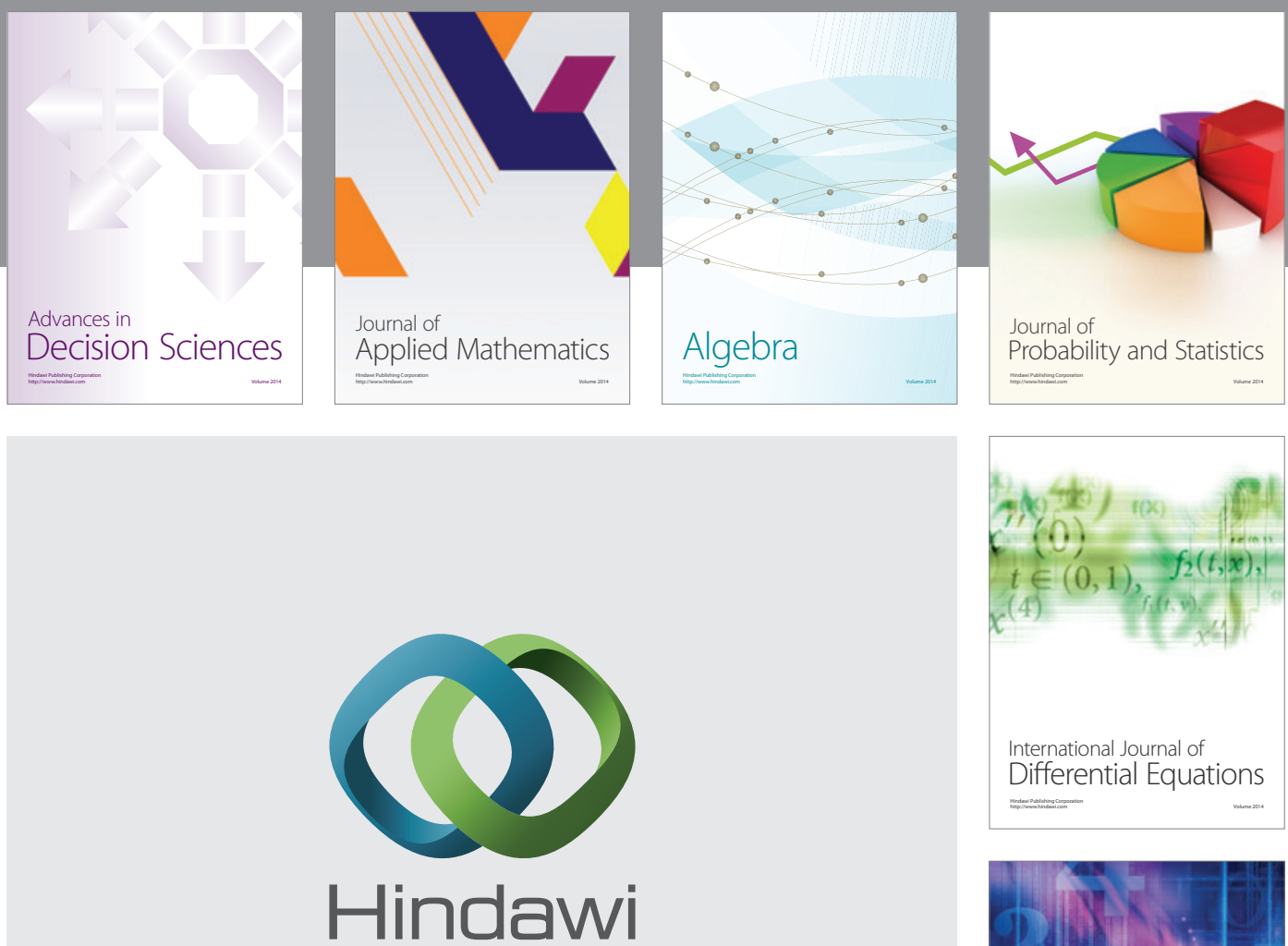

Submit your manuscripts at http://www.hindawi.com
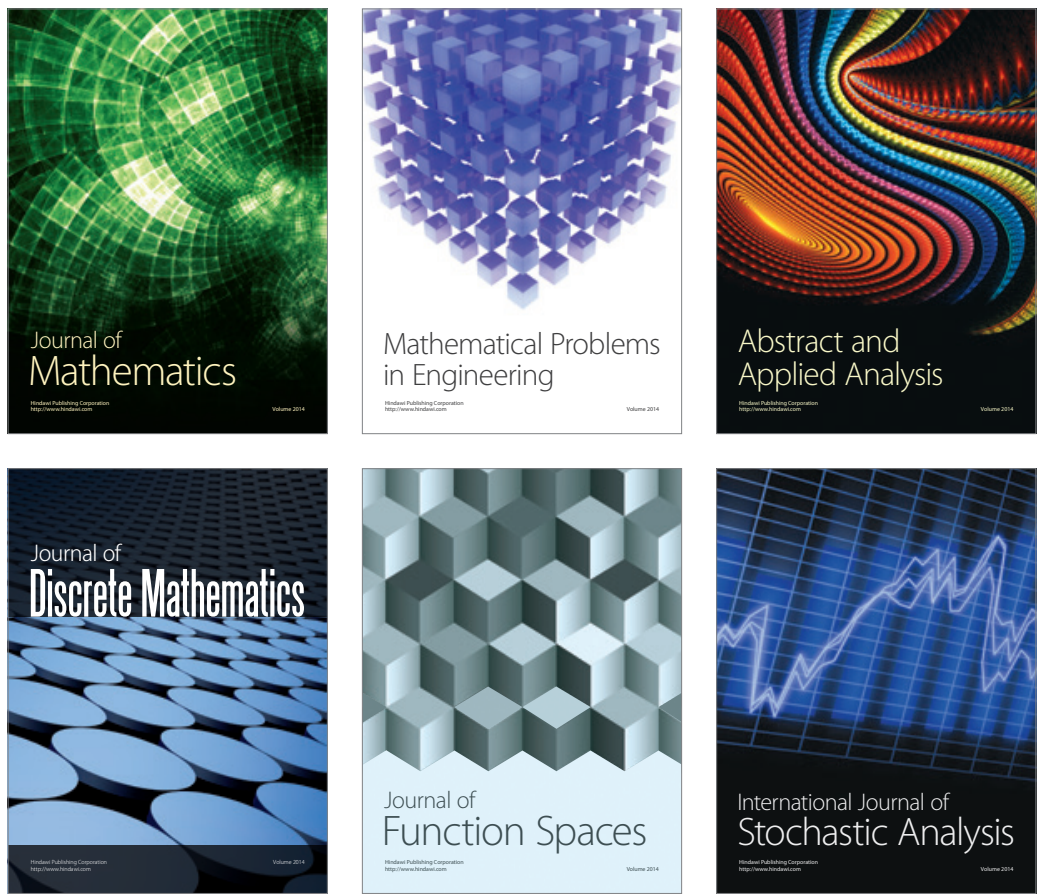

Journal of

Function Spaces

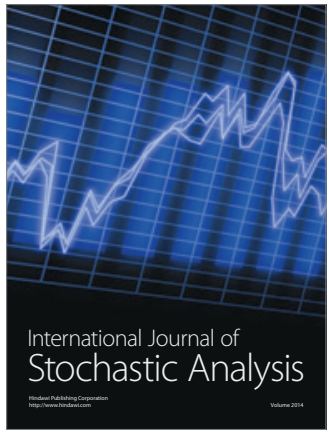

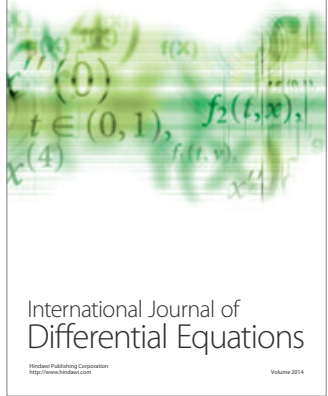
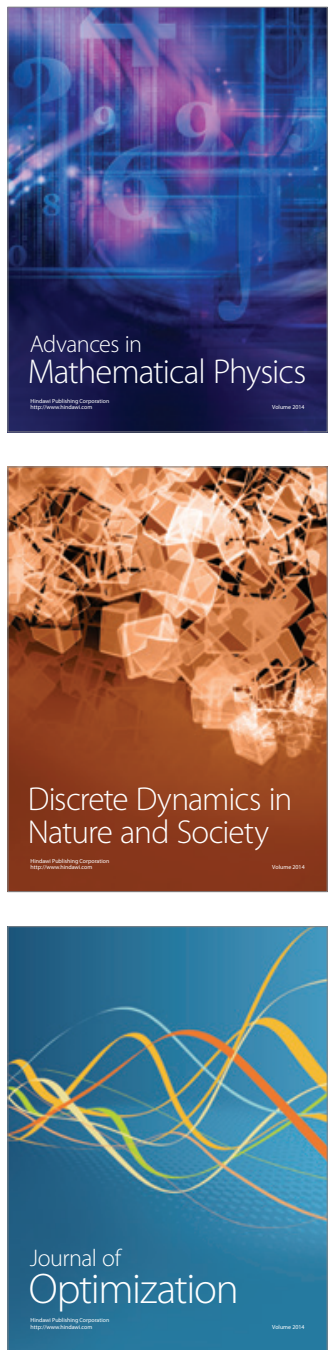